\title{
Zytologische Untersuchungen an „Mitochondrienfraktionen“ von Hefen
}

\author{
Von Elisabeth Bautz \\ Aus dem Forstbotanischen Institut der Universität Freiburg i. Br. * \\ (Z. Naturforschg. 11 b, 26-31 [1956]; eingegangen am 27. September 1955)
}

\begin{abstract}
Die Fraktion der mikroskopisch sichtbaren Plasmapartikel von Hefen, welche einer biochemischen Mitochondrienfraktion entspricht, wurde eingehend zytologisch untersucht. Mit Hilfe des Phasenkontrast-Verfahrens und verschiedener Färbemethoden konnten wir in solchen Fraktionen Mitochondrien, Sphärosomen, Zellkerne, Membranen und Plasmakoagulationen oder Gerinnsel identifizieren. Es gelang weiterhin, diese verschiedenen Sorten von Plasmabestandteilen durch fraktioniertes Zentrifugieren in Unterfraktionen anzureichern.
\end{abstract}

$\mathrm{B}_{\mathrm{t}}^{\mathrm{e}}$ ei den biochemischen Arbeiten an aufgearbeiteten (homogenisierten) Zellen ging man ganz allgemein von der Annahme aus, daß die Zelle außer dem Zellkern und evtl. den Plastiden bei Pflanzenzellen nur eine Sorte von mikroskopisch sichtbaren Zytoplasmapartikeln, die Mitochondrien, besitzt ${ }^{1,2,3}$. Somit wurde von den verschiedensten Autoren angenommen, daß die durch differenziertes Zentrifugieren gewonnene Fraktion von mikroskopisch sichtbaren Plasmapartikeln, die Mitochondrienfraktion, tatsächlich aus einheitlichen Zytoplasmapartikeln besteht. Diese Meinung wurde vor allem auch durch zytologische Untersuchungen an Mitochondrienfraktionen bei lichtmikroskopischer Prüfung von Präparaten, die mit dem typischen Mitochondrienfarbstoff, Janusgrün ${ }^{3}$, gefärbt waren, bestärkt, denn es zeigen sich in den meisten Fällen tatsächlich ausschließlich grün angefärbte Bestandteile ${ }^{4,5,6}$.

So ist es zu verstehen, daß man die an „Mitochondrienfraktionen “ gewonnenen biochemischen Ergebnisse ohne weiteres den Mitochondrien zuschrieb.

Wir kennen aber auch aus der biochemischen Literatur warnende Stimmen, die eindringlich darauf hinweisen, daß die Mitochondrienfraktion keineswegs einheitlich sein müsse (z. B. L a n g ${ }^{7}$ ).

- Durchführung der Untersuchungen im Physiolog.Chem. Institut der Universität Mainz. Herrn Prof. Dr. Dr. K. L a ng, Direktor des Physiol.-Chem. Instituts der Universität Mainz, danke ich für die Ermöglichung dieser Arbeit.

1 O. Lindberg u. L. Ernster, Protoplasmatologia III, Wien 1954 .

2 A. Goddard u. H. Stafford, Annu. Rev. Plant. Physiol. 5, 115 [1954].

3 A. Millerd u. J. B o n ner, Histo-Cytochem. 1, 254 [1953].

4 R. W. Chessin, zit. b. M. Gers ch, Erg. mod. Biol. 31, 57 [1951].
Aus zytologischen Untersuchungen an intakten, lebenden und fixierten Zellen von höheren Pflanzen wissen wir nun, daß es außer den Plastiden 2 weitere, voneinander streng verschiedene Sorten von Zytoplasmapartikeln gibt, die Mitochondrien und Sphärosomen (Perner ${ }^{8,9}$, D rawert ${ }^{10}$, S oro kin $\left.{ }^{11}\right)$. Dieselben Verhältnisse fanden wir auch bei unseren untersuchten Pilzen wie z.B. den Hefen (B a u t $\mathrm{z}^{12,13}$, M a r quard t und B a u t ${ }^{14}$ ). Nachdem wir nun in den letzten Jahren die Plasmapartikel an lebenden und fixierten Hefezellen eingehend studiert hatten und die Mitochondrien und Sphärosomen in ihrer Lichtbrechung unter den verschiedenen Bedingungen sowie auch das färberische Verhalten dieser Partikel kennenlernten, war es unser Ziel, die sogenannte Mitochondrienfraktion von Hefen zytologisch zu prüfen.

\section{Material und Methode}

Für unsere Untersuchungen verwendeten wir die Bäckerhefe Saccharomyces cerevisiae Nr. 365, die uns freundlicherweise von der Firma Sinner A.G. Karlsruhe als Reinkultur in beliebiger Menge zur Verfügung gestellt wurde. Die Hefen wurden in 8,5-proz. Rohrzuckerlösung aufgeschwemmt und gewaschen, dann nach kurzer Zentri-

${ }^{5}$ A. Claude u. F. Full a m, J. Exp. Medicine 81, 51 [1945].

6 A. Millerd et al., Proc. Nat. Acad. Sci. (USA) 37,855 [1951].

7 K. L a n g, Koll. Ges. Physiol. Chemie, Berlin 1952.

8 E. S. Perner, Biol. Zbl. 71, 43 [1952].

9 E. S. P e rn er, Protoplasma 42, 457 [1953].

$10 \mathrm{H}$. D $\mathrm{raw}$ ert, Ber. dtsch. bot. Ges. 66, 13.5 [1953].

11 H. Sorokin, Amer. J. Bot. 42, 225 [1955].

1. E. B a u tz, Naturwissenschaften 42, 49 [1955].

13 E. B a utz, Z. Bot., im Druck (Sammelreferat).

14 H. M a rquardt u. E. B a utz, Arch. Mikrobiol., im Druck. 
fugation in frischer Zuckerlösung als 10-proz. Suspension zur weiteren Untersuchung verwendet. Wir bemühten uns weitgehend, steril zu arbeiten.

Die Zerkleinerung der Hefezellen, vor allem das Aufbrechen der derben Hefemembranen, erfolgte durch mechanische Zertrümmerung mit Hilfe einer Schüttelmaschine, welche von Herrn Ing. K ö h ler, physiol. Inst. der Universität Mainz, nach einem von N o s s a 115 beschriebenen Apparat entwickelt worden war. In dieser Maschine schüttelten wir $50 \mathrm{ccm}$ unserer 10-proz. Hefesuspension mit $450 \mathrm{~g}$ Stahlkugeln $(2-3 \mathrm{~mm}) 1 \mathrm{Min}$. lang in einer Metallbüchse bei etwa 6750 Touren/Minute. Die vorgekühlte Hefesuspension wurde während dieser kurzen Zertrümmerungszeit von $5^{\circ} \mathrm{C}$ auf etwa $30^{\circ} \mathrm{C}$ erwärmt. Bei mikroskopischer Kontrolle stellten wir danach 40-60\% aufgeplatzte Hefezellen fest.

Die geschüttelten Hefesuspensionen wurden anschlieBend fraktioniert zentrifugiert. Zunächst mit einer Zentrifuge bei $1750 \mathrm{~T}=650 \mathrm{~g}$ ( $\mathrm{g}=$ Erdbeschleunigung), danach die überstehende Flüssigkeit erneut bei $3000 \mathrm{~T}=$ $2000 \mathrm{~g}$.

Der Überstand kam nun zur weiteren Zentrifugation bei $15000 \mathrm{~T}$ der Hochleistungszentrifuge, welche etwa $14800 \mathrm{~g}$ entspricht. Diese letzten Zentrifugationen mußten in einem Kühlraum durchgeführt werden.

Zur zytologischen Darstellung der Zellkerne wurden die Fraktionen der isolierten Partikel nach Feulgen gefärbt ( $\mathrm{R} \circ \mathrm{m}$ e is 16). Die Fixierung erfolgte in Carnoy (3 Tle. Alkohol 96\% und $1 \mathrm{Tl}$. Eisessig), hvdrolysiert wurde $10 \mathrm{Min}$. lang in 1-n. Salzsäure bei $60^{\circ} \mathrm{C}$. Die Färbung dauerte $3 \mathrm{Stdn}$. in fuchsinschwefliger Säure und die Differenzierung mit $\mathrm{SO}_{2}$-haltiger Spülflüssigkeit 6 bis 8 Minuten.

Für die Färbung von Mitochondrien verwendeten wir die Tanusgrün B-Färbung ( $\mathrm{S}$ or o k i n ${ }^{17}, \mathrm{~B}$ a u t $\mathrm{z}^{18}$ ). 1 bis 10 Tropfen einer 1-proz. Stammlösung von Janusgrün B Grübler wurde zu $50 \mathrm{ccm}$ der Rohrzuckerlösung gegeben. Wir ließen diese Lösungen 2 bis 3 Stdn. lang einwirken. Zum Nachweis der Plasmagranula oder Sphärosomen verwendeten wir die Nadi-Reaktion nach P e r n e r : $2 \mathrm{ccm}$ a-Naphthol und 2 ccm $p$-Phenylendiamin (beides $0,1 \%$, wäßrig gelöst) wurden zu $6 \mathrm{ccm}$ Phosphatpuffer vom $p_{\mathrm{H}}$ 6.8 gegeben. Die Färbungszeit dauerte 30 und u. U. 50 Minuten ( $\mathrm{B}$ a u t $\mathrm{z}^{18}, \mathrm{M}$ a r q u a r d $\mathrm{t}$ und $\mathrm{B}$ a u $\mathrm{z}^{19}$ ).

Optik: Zeiss-Opton Stativ W mit Phasenkontrast-Einrichtung (100X Ölimmersion, Optovar $1 \times$, Okular $8 \times$ ). Für Mikroaufnahmen stand eine Leica mit Mikroaufsatzgerät zur Verfügung.

\section{Ergebnisse}

A. Die Partikel der, Mitochondrienfraktion" von Hefen

Um über die uns interessierenden, verschiedenen Plasmapartikel der Hefezelle im Bilde zu sein, ha-

15 R. M. N o s s a l, Austral. J. exp. Biol. med. Sci. 31, 583 [1953].

16 B. R o m e is, Mikroskop. Technik, München 1948.

17 H. S or ok in, Amer. J. Bot. 25, 28 [1938]. ben wir in Abb. $1^{*}$ eine intakte, lebende Hefezelle schematisch dargestellt. Abb. 2 a und b zeigen die im Phasenkontrast sichtbaren Mitochondrien und weniger gut zu erkennende, in einer anderen Ebene des mikroskopischen Bildes liegende Sphärosomen. In Abb. $3 \mathrm{a}$ und $3 \mathrm{~b}$ sind dagegen allein die Sphärosomen deutlich zu sehen.

Zur Gewinnung einer sogenannten „Mitochondrienfraktion " von Hefen wurden Hefe-Suspensionen mit $50 \%$ mechanisch zertrümmerten Hefen bei verschiedener Tourenzahl differenziert zentrifugiert. Bei eine ${ }_{i}$ 3 Min. dauernden Zentrifugation mit $650 \mathrm{~g}$ gelang es fast vollständig, die unbeschädigten Hefezellen abzutrennen, danach wurde die überstehende trübe Lösung bei $6000 \mathrm{~g}$ erneut 3 Min. lang zentrifugiert. In dem nun gewonnenen Sediment fanden wir aufgeplatzte Zellhüllen (Abb. 4), z. T. auch größere abgekugelte Plasmaschollen, Zellfetzen und Plasmagerinnsel, u. U. auch Verunreinigungen, die anscheinend trotz des Waschens der Hefe-Suspension mit in die Lösung gelangt waren.

Anschließend zentrifugierten wir die überstehende Lösung in der Hochleistungszentrifuge bei $14800 \mathrm{~g}$ etwa $30 \mathrm{Min}$. lang. Wir erhielten auf diese Weise die Fraktion der mikroskopisch sichtbaren Plasmapartikel, die einer biochemischen Mitochondrienfraktion entspricht (Abb. 5). Der Überstand der ,Mitochondrienfraktion “wurde nun erneut $30 \mathrm{Min}$. bei $14800 \mathrm{~g}$ zentrifugiert. In dieser weiteren Fraktion konnten wir aber selbst bei phasenoptischer Betrachtung keinerlei Plasmapartikel mehr genau erkennen, bestenfalls sah man noch kleines, schwach lichtbrechendes PlasmaGerinnsel.

Wir unterzogen nun die sedimentierten Plasmapartikel der sog. Mitochondrienfraktion einer eingehenden zytologischen Prüfung. Bei lichtmikroskopischer Beobachtung konnten wir lediglich graues Gerinnsel und dunkelgrau-schwarze Granula erkennen.

Erst durch die Untersuchungen mit Phasenkontrast erhielten wir durch die verschiedene spezifische Lichtbrechung der einzelnen Plasmabestandteile ein genaueres Bild. Zunächst fielen uns die deutlich schwarzen, stark lichtbrechenden Granula auf, die sich mit den Sphärosomen der intakten Zelle durchaus vergleichen ließen, weiterhin sah man größere runde, mehr violett erscheinende Gebilde, die meist einen dunkleren, z. T. sichel- oder napfförmigen

1s E. B a utz, Ber. dtsch. bot. Ges. 68, 197 [1955].

19 H. Marquardt u. E. B a u tz, Arch. Mikrobiol., im Druck.

* Abb. 1-9 s. Tafel S. 32 a. 
Rand besaßen. Durch die spezifische Lichtbrechung konnte man diese Gebilde mit den Mitochondrien der intakten Zelle vergleichen, vor allem aber mit Mitochondrien von geschädigten Zellen, am besten von Hefen nach Röntgenbestrahlung. Außerdem gab es noch schwach lichtbrechende Plasmaschollen und mehr oder weniger stark lichtbrechendes Plasmagerinnsel, welches auch etwas dunklere Stellen besaß, sowie stark lichtbrechende Gebilde, größer als die Sphärosomen. Diese stark lichtbrechende Strukturen lösen sich in Xylol vollständig auf und werden deshalb vermutlich Fett-Tropfen sein, die bei der Aufarbeitung der Hefen entstanden sein mußten. Man sah außerdem noch schwarze Zellmembranen, graue Plasmahäutchen- und -schollen in dieser Fraktion. In Abb. 6 sind die in der Mitochondrienfraktion identifizierten Partikel zeichnerisch dargestellt.

Um exaktere Aussagen über diese beobachteten Strukturen machen zu können, wandten wir die für die verschiedenen Sorten von Plasmapartikeln spezifischen Färbemethoden an.

Zunächst färbten wir nach Feulgen, denn es ist uns aus Untersuchungen an intakten fixierten Hefezellen bekannt, daß der Zellkern der Hefe, etwa in der Größenordnung der Zytoplasmapartikel liegt (Abb. 7). Mit dieser Feulgenfärbung wurden ausschließlich runde oder $u$. $U$. etwas länglichere Gebilde angefärbt, die kaum größer als die stark lichtbrechenden Plasmagranula sind (Abb. 8). Um nun die Plasmagranula, welche wir als Sphärosomen ansahen, näher identifizieren zu können, unterzogen wir die Mitochondrienfraktion einer Nadi-Reaktion, mit welcher bei normal atmenden, intakten Hefen stets die Sphärosomen angefärbt wurden. Nach der für lebende Hefezellen günstigen Einwirkungszeit von 30 Min. zeigte sich bei lichtmikroskopischer Kontrolle der Suspension, daß sich zunächst die stark lichtbrechenden Granula indophenolblau gefärbt hatten, wie wir es von den Sphärosomen der intakten Hefen her kennen. Aber außer solchen Sphärosomen färbten sich noch die großen stark lichtbrechenden Strukturen und weiterhin noch kleine Punkte im Plasmagerinnsel, welche sich in Xylol lösen und darum vermutlich Fett-Tropfen darstellen. Wir konnten dagegen in keinem Fall beobachten, daß sich die schwach lichtbrechenden runden oder auch mehr aufgequollenen ringförmigen Gebilde anfärbten, die wir im Phasenkontrast als Mitochondrien identifizierten.

Um nun die Mitochondriennatur dieser Partikel nachzuweisen, färbten wir die Fraktion mit Janusgrün $\mathrm{B}$, welches ja durch die bei Sauerstoffabschluß eintretende Entfärbung als typische Mitochondrienfärbung gilt. Wir verwendeten zunächst die bei früheren Untersuchungen an lebendem Zellmaterial günstigen Lösungen ${ }^{20}$ und erhielten dabei keine Färbung. Erst bei etwas stärkeren Konzentrationen (8 Tropfen der Stammlösung anstatt $1-2$ zu $50 \mathrm{ccm}$ Versuchslösung) färbten sich nach $2 \mathrm{Stdn}$. Einwirkungszeit sowohl die Plasmaschollen und Plasmagerinnsel als auch die Sphärosomen grünlich und die vermutlichen Mitochondrien schwach an. Nach Sauerstoffabschluß blieb diese Färbung unverändert erhalten. In sehr seltenen Fällen fanden wir aber einzelne dieser Mitochondrien, oftmals nur den sichelförmigen Rand, mit Janusgrün B tiefer blaugrün angefärbt. Nach $30 \mathrm{Min}$. bis 1 Stde. Sauerstoffabschluß durch Paraffinumrandung des Präparates blaßten diese blaugrün gefärbten Strukturen deutlich ab.

Durch diese Farbreaktion, Blaugrünfärbung und Abblassen der Farbtiefe bei Sauerstoffmangel, glauben wir nachgewiesen zu haben, daß die Gebilde tatsächlich Mitochondrien sind, denn auch in lebenden Zellen verhalten sich die Mitochondrien gegenüber Janusgrün B in gleicher Weise.

Bei unseren zytologischen Untersuchungen an $\mathrm{Mi}$ tochondrienfraktionen fanden wir in einigen Fällen Bakterien-Infektionen. Obwohl wir immer versucht hatten, steril $\mathrm{zu}$ arbeiten, gelangten in die Mitochondrienfraktion oftmals auch einzelne kokkenförmige Bakterien, die in der Größe den Mitochondrien gleichen, aber homogen und stärker lichtbrechend sind. Sie färbten sich meist mit Janusgrün B an, ohne sich aber bei Sauerstoffentzug zu entfärben. Ließ man die Fraktionen einige Stdn. im Eisschrank stehen, so waren Bakterien in großer Anzahl vorhanden. Für längeren Zeitraum beanspruchende, biochemische Fermentnachweise dürften diese kaum zu vermeidenden Bakterien-Infektionen ein schwerwiegendes Problem bedeuten.

\section{B. Versuch einer Trennung der ver- schiedenen in der, Mitochondrien- \\ fraktion" vorkommenden Sorten von Plasmapartikeln}

Nachdem wir die verschiedenen Zellbestandteile. Kerne, Mitochondrien und Sphärosomen, in der „,Mitochondrienfraktion" identifiziert hatten, versuchten wir durch sehr vorsichtige und kurze Zentrifugierungen. die einzelnen Zellbestandteile voneinander zu trennen oder doch wenigstens in verschiedenen Fraktionen anzureichern.

0ㅡ E. B a u z , Ber. dtsch. bot. Ges. 68, 197 [1955]. 
Mit der zur Verfügung stehenden Hochleistungszentrifuge, welche auf 15000 Touren, d. h. $14800 \mathrm{~g}$, eingestellt war, und mit deren Hilfe wir bisher die sogenannte Mitochondrienfraktion hergestellt hatten, besaßen wir die Möglichkeit, durch verschieden langes Zentrifugieren die „Mitochondrienfraktion“ in verschiedene Unterfraktionen aufzuteilen. So erhielten wir an Stelle einer „Mitochondrienfraktion“ 4 verschiedene Fraktionen (Sedimente 1-4).

Nach 8 Sek. Zentrifugieren bei $14800 \mathrm{~g}$ erhielten wir eine Fraktion mit hauptsächlich Plasmaschollen, kleinen Portionen von Plasmagerinnseln mit einigen Sphärosomen, Mitochondrien und sehr wenigen Zellkernen. In dieser Fraktion fanden wir außerdem noch freiliegende Sphärosomen, Kerne, zarte Plasmafetzen und wenige Mitochondrien, evtl. sogar noch Verunreinigungen. Der Überstand der eben beschriebenen Fraktion wurde danach nochmals 1 Min. lang bei $14800 \mathrm{~g}$ zentrifugiert. Dieses 2. Sediment enthielt freiliegende Sphärosomen, u. U. mit etwas anhaftendem Zytoplasma, dann auch Mitochondrien, seltener Zellkerne (Abb. 9) und auch einzelne FettTropfen. Nach erneutem Zentrifugieren des Überstandes entnahmen wir nach $30 \mathrm{Min}$. ein 3. Sediment, es bestand aus Sphärosomen und Mitochondrien, etwa beide in derselben Häufigkeit, ferner auch aus den schon in Sediment 2 aufgetretenen Fett-Tropfen, manchmal wenigen, abgekugelten Bakterien-Infektionen. Zentrifugierten wir die bei Sediment 3 überstehende ziemlich klare, gelbliche Lösung 1 Stde. lang bei $14800 \mathrm{~g}$, so entdeckten wir in diesem Sediment 4 erst nach phasenoptischer Betrachtung kleinste, stärker und schwächer lichtbrechende, an der Grenze des mikroskopisch Sichtbaren liegende Partikelchen, die aber nicht mehr zu identifizieren waren.

Überblicken wir nun die Ergebnisse unserer „Trennungsversuche“ der Mitochondrienfraktion, so zeigt sich, wie es eigentlich von vorneherein auch zu erwarten war, daß es bei den zur Verfügung stehenden technischen Möglichkeiten nicht gelang, die ein-

21 A. Claude u. F. Full am, J. Exp. Medicine 81, 51 [1945].

22 R. W. Ches s in, zit. b. M. Gers ch, Erg. mod. Biol. 31, 57 [1951].

23 J. M c Clendon, Amer. J. Bot. 39, 275 [1952].

24 O. Lindberg u. L. Ernster, Protoplasmatologia III, Wien 1954.

25 K. L a n g, Koll. Ges. Physiol. Chemie, Berlin 1952.

26 A. M illerd u. J. B o n n er, Histo-Cytochem. 1, 254 [1953].

27 A. Goddard u. H. St a fford, Annu. Rev. Plant Physiol. 5, 115 [1954]. zelnen Sorten von Plasmapartikeln auch nur annähernd voneinander zu trennen. Trotz dieser Hindernisse konnten wir aber feststellen, daß das Vorkommen von isolierten Mitochondrien bei unseren Versuchsbedingungen in den Sedimenten 1 bis $3 \mathrm{zu}$ nahm.

\section{Diskussion}

Claude und Fullam²1, Chessin², McClendon ${ }^{23}$ u. a. hatten zytologische Untersuchungen an Mitochondrienfraktionen der Gewebe von Tieren und höheren Pflanzen unternommen. Bei solchen Färbeversuchen wurde die Janusgrün-Färbbarkeit der isolierten Plasmapartikel als Beweis für ihre Mitochondriennatur angesehen. Auf diesem Wege gelangte man zu der Überzeugung, daß die mit Janusgrün gefärbten Partikel der Mitochondrienfraktion tatsächlich Mitochondrien sind; die in dieser sogenannten Mitochondrienfraktion nachgewiesenen Fermente, wie Atmungsfermente, Fermente der Phosphorylierung und auch solche für Synthesen, ebenso der nachgewiesene RNS-Gehalt wurden ganz allgemein den Mitochondrien zugeschrieben (Zusammenfassungen bei Lin dberg und Ernster ${ }^{24}$, Lang ${ }^{25}$, Millerd und B on ne r ${ }^{26}$, G odd a rd und $S \mathrm{taf}$ f or ${ }^{27}$ ).

Auch bei Untersuchungen und den Fermentnachweisen an Hefen faßte man die mikroskopisch sichtbaren Plasmapartikel in einer einzigen Fraktion zusammen (z. B. Slonimski ${ }^{28}$, Nossal ${ }^{29}$ u. a.), verzichtete aber auf eine genauere zytologische Untersuchung dieser Fraktionen.

Aus eingehenden zytologischen Studien an lebenden Pflanzen-Zellen wissen wir durch die Untersuchungen von Sorokin ${ }^{30}$, D rawe rt ${ }^{31}$, Perner und Pfefferkorn ${ }^{32}$ an Allium cepa, Bautz ${ }^{33}$, $\mathrm{M}$ a rquardt und $\mathrm{B}$ a u $\mathrm{Z}^{34}$ an Hefen und an weiteren Pilzen von $\mathrm{B} \mathrm{autz}{ }^{33}$, daß es 2 verschiedene Sorten von Zytoplasmapartikeln, die Mitochondrien und Sphärosomen, gibt und daß Janusgrün $\mathrm{B}$ bei geschädigten Zellen nicht als Mitochondrienfarbstoff zu werten ist, da sich unter diesen Umständen alle

28 P. P. S lonimski, Actualites Biochimiques, Paris 1953.

29 R. M. N o s s a l, Austral. J. exp. Biol. med. Sci. 31, 583 [1953].

30 H. S or ok in, Amer. J. Bot. 42, 457 [1955].

31 H. D r a w ert, Ber. dtsch. bot. Ges. 66, 135 [1953].

32 E. Perner u. G. Pfefferkorn, Flora 140,98 [1953].

33 E. B a utz, Z. Bot., im Druck.

34 H. M a rquardt u. E. B a u tz, Arch. Mikrobiol., im Druck.

35 R. B uvat, Endeavour 12, 33 [1953]. 
Plasmapartikel, sogar auch das Zytoplasma selbst, anfärben. Wir dürfen deshalb als Kriterium für eine echte Mitochondrienfärbung mit Janusgrün B allein die bei Sauerstoffmangel eintretende Entfärbung der Mitochondrien betrachten. Durch diesen spezifischen Färbungsmodus können wir auch die echten" Mitochondrien ohne weiteres von den u. U. unspezifisch mit Janusgrün B angefärbten Sphärosomen unterscheiden, die sich ja bei der Nadi-Reaktion mit Indophenolblau färben.

Wir haben also an lebenden Zellen ohne weiteres die Möglichkeit, die 2 verschiedenen Sorten von Zytoplasmapartikeln mit Hilfe verschiedener Lebend-Färbungen und außerdem phasenoptisch durch die unterschiedliche Lichtbrechung voneinander zu unterscheiden. Sobald aber die Zelle durch irgendwelche äußere Einwirkungen geschädigt wird, finden wir eine Abkugelung und ein Blasigwerden der Mitochondrien ${ }^{35}$, und die Janusgrünfärbung wird unspezifisch. Lediglich die Sphärosomen bleiben in ihrer Form unverändert erhalten und färben sich weiterhin elektiv mit dem Nadi-Reagens ${ }^{36}$. P e r n e r und $\mathrm{Pfefferkorn}{ }^{37}$ haben an geschädigten und aufgebrochenen Allium-Epidermen eine genauere zytologische Analyse unternommen und kamen zu denselben Befunden. Diese Autoren stellten weiterhin fest, daß durch die Zellschädigung auch Plasmagranulationen und Plasmaschollen entstanden waren.

Nach diesen Ergebnissen an geschädigten oder verletzten Zellen war es zu erwarten, daß unsere durch mechanische Zertrümmerung und für die Gewinnung von "Mitochondrienfraktionen " bei hohen Touren zentrifugierten Plasmabestandteile der Hefen sich in ihrem spezifischen Verhalten verändert haben mußten. Bei genauerer zytologischer Untersuchung fanden wir in der „Mitochondrienfraktion “ sehr verschiedene Plasmabestandteile. Mit der Feulgenfärbung konnten wir runde Zellkerne nachweisen, die stark lichtbrechenden und ziemlich unveränderten Sphärosomen färbten sich weiterhin mit der Nadi-Reagens. Die Mitochondrien konnte man zunächst im Phasenkontrast-Mikroskop an ihrer schwachen Lichtbrechung vermuten, in ihrer Form hatten sie sich z. T. stark verändert. Die in der lebenden Zelle stäbchenförmigen bis runden Mitochondrien waren in der Fraktion

36 E. B a u z , Z. Bot., im Druck (Sammelreferat).

37 E. Perner u. G. Pfefferkorn, Flora 140 , 98 [1953].

38 A. La a z a row u. S. Cooperstein, Exp. Cell Res. 5, 56 [1953].

39 Ph. Hartman u. Ch. Liu, J. Bacteriol. 67, 77 [1954]. alle abgekugelt, z. T. aufgequollen und blasig oder auch sichel- und ringförmig. Mit Janusgrün färbten sich die meisten dieser Mitochondrien sehr schwach grünlich und in seltenen Fällen auch blaugrün, nach Sauerstoffabschluß blaßten aber allein die blaugrün gefärbten $a b$ und verhielten sich somit etwa wie die „aktiv atmenden“ Mitochondrien. Es ist möglich, daß die sich nicht mehr entfärbenden Mitochondrien stärker geschädigt worden sind als die noch typisch für Mitochondrien reagierenden, oder daß ihre Fermente während der langen Fraktionierungs- und Färbedauer abgebaut worden sind. - Mit den von uns verwendeten, recht schwachen Konzentrationen von Janusgrün B färbten sich aber weiterhin die Sphärosomen, Zellkerne, Zellmembranen, Plasmagerinnsel und Plasmaschollen in verschiedener Intensität an.

Unsere Ergebnisse zeigen also, daß Janusgrün B außerhalb der lebenden Zelle alle aus Eiweißstoffen bestehenden Partikel unspezifisch anfärbt, wie es nach den chemischen Untersuchungen von $\mathrm{L}$ a a $\mathrm{z}$ a row und C o o per s te in ${ }^{38}$ über den Mechanismus der Janusgrünfärbung zu erwarten war. Es bedarf deshalb einer genauen Kenntnis und des Studiums der Mitochondrien, um sie in den biochemischen Fraktionen auf Grund von Lichtbrechung, Form und Färbbarkeit wieder zu erkennen.

Dasselbe gilt, freilich in etwas anderem Sinne, auch für die Sphärosomen. Zwar sind sie gegenüber den Eingriffen bei der Fraktionierung wesentlich resistenter und verändern sich gestaltlich nur wenig, aber bei der Indophenolblau-Färbung, die wir zu ihrer Charakterisierung verwenden, treten ebenfalls Schwierigkeiten auf. Während P e r n e r ${ }^{43}$ bei pflanzlichen Zellen die Färbung bestimmter Plasmapartikel durch Indophenolblau als beweisend für dort lokalisierte, Sauerstoff umsetzende Fermente ansieht, ist es nach Arbeiten von D i e t r i c h ${ }^{40}, \mathrm{Z}$ i e g l e r ${ }^{41}$, D r a w e r t ${ }^{42}$ u. a. ebenso möglich, daß der Farbstoff von lipoidreichen Partikeln gespeichert wird.

Übereinstimmend mit diesen Befunden färben sich in unseren Fraktionen außer den Sphärosomen auch die wesentlich größeren Fett-Tropfen, welche mit Xylol verschwinden. Es können daher indophenolblau-gefärbte Partikel in einer Fraktion nicht ohne weiteres als Sphärosomen angesprochen werden; ersí die Berücksichtigung ihrer Größe, ihres Verhaltens gegenüber fettlösenden Mitteln und ihr Verhalten be-

40 A. D i etrich, Zbl. Pathol. 19, 3 [1908].

41 H. Z i e g l e r, Z. Naturforschg. 8 b, 662 [1953].

42 H. D r a w e r t, Ber. dtsch. bot. Ges. 66, 135 [1953].

43 E. S. P erner, Biol. Zbl. 71, 43 [1952]. 
züglich Lichtbrechung läßt uns die wahren Sphärosomen unterscheiden von anderen, lipoidreichen und darum farbspeichernden Strukturen.

Da nach allen vorliegenden Erfahrungen die Sphärosomen lipoidreiche Partikel darstellen, ist die vor allem von Drawert ${ }^{42}$ und uns $\left(\mathrm{Bautz}^{36}\right)$ geäußerte Auffassung durch die hier vorgelegten Befunde gestützt, daß möglicherweise die Atmungsfermente in den ,echten “ Mitochondrien lokalisiert sind, die wir mit Janusgrün B anfärben und die nach Sauerstoffentzug wieder entfärbt werden; die Sphärosomen würden demnach im wesentlichen nur infolge ihres Lipoidreichtums sekundär das gebildete Indophenolblau speichern.

Vor allem aus diesen experimentell noch nicht ausreichend gestützten Überlegungen folgt unmittelbar, welche besondere Bedeutung einem Verfahren zukäme, durch welches einerseits eine reine Mitochondrienfraktion, andererseits eine reine Sphärosomen- fraktion hergestellt werden könnte, um ihre jeweilige stoffliche bzw. fermentative Zusammensetzung zu prüfen. Unsere hier vorgelegten Untersuchungen stellen den ersten Schritt in dieser Richtung dar und haben als wesentliches Ergebnis erbracht, daß tatsächlich die Mitochondrien bei unserem Versuchsobjekt Saccharomyces cerevisiae (Sinner 365) und unseren Versuchsbedingungen etwas später sedimentieren als die Sphärosomen. Auf der anderen Seite werden sich diese beiden Sorten von Plasmapartikeln wohl kaum vollständig voneinander trennen lassen, da sie sich wahrscheinlich physikalisch zu wenig voneinander unterscheiden; als weitere Schwierigkeiten kommen das Vorhandensein des nur schwer zu entfernenden, etwa die Größe der Zytoplasmapartikel besitzenden Zellkerns sowie die Anwesenheit von Plasmaresten hinzu, die den Mitochondrien und Sphärosomen anhaften oder von ihnen aus anderen Gründen nicht zu trennen sind.

\title{
Die Änderung der serologischen Spezifität von Leberzellen der Ratte während der Cancerogenese durch $p$-DimethylaminoazobenzoI
}

\author{
Von Eberhardt Weiler \\ Aus dem Max-Planck-Institut für Virusforschung, Tübingen \\ (Z. Naturforschg. 11 b, 31-38 [1956]; eingegangen am 25. Juli 1955)
}

\begin{abstract}
Mit Hilfe leber-spezifischer fluorescein-gekuppelter Antikörper konnte histo-serologisch bestätigt werden, daß im primären Leberzellkarzinom der Ratte das leber-spezifische Antigen nicht nachweisbar ist.

Das leber-spezifische Antigen ist ausschließlich in den Leberparenchymzellen lokalisiert.

Lebergewebe, das unter dem Einfluß des Cancerogens gestanden hatte, enthält weniger leber-spezifisches Antigen als normale Leber. Das äußert sich im Komplementbindungs-Test in einer verminderten leber-spezifischen serologischen Aktivität der Mikrosomen und Mitochondrien und histo-serologisch im Auftreten von charakteristischen Gewebeinseln, in denen die Antigenkonzentration gegenüber dem umliegenden Gewebe vermindert ist.

Der Grad der Antigenverarmung ist abhängig von der verfütterten Gesamtdosis des Cancerogens und von der Latenzzeit.
\end{abstract}

$\mathrm{D}$ e Mikrosomen- und Mitochondrien-Fraktionen von Leberzellen der Ratte enthalten ein organspezifisches Antigen ( $\mathrm{Furth}, \mathrm{H}$ en le $\left.{ }^{1,2}\right)$. Frühere Versuche (Weile ${ }^{3,4}$ ) hatten gezeigt, daß dieses organspezifische Antigen in cancerisierten Zellen, also im primären hepatocellulären Carcinom, nicht mehr nachweisbar ist. Das Ziel der vorliegenden Arbeit war es, das Verhalten des leber-spezifischen Antigens

1 J. Furth u. E. A. Kabat, J. exp. Medicine 74, 257 [1941].

2 W. Henle, L. A. Chambers u. V. Groupe, J. exp. Medicine 74, 495 [1941]. während der Behandlung der Ratten mit $p$-Dimethylaminoazobenzol (DAB) genauer zu verfolgen. Die DAB-Gesamtdosis, die Tagesdosis und die Latenzzeit (= Zeit nach Aufhören der DAB-Fütterung bis zur Tötung der Tiere) wurden dabei variiert.

Für die serologische Untersuchung standen zwei Methoden zur Verfügung:

Die durchschnittliche Antigenmenge konnte durch Austesten der Mikrosomen- und Mitochondrienfraktionen mit leber-spezifischem Antiserum im Komple-

3 E. W e il e r, Z. Naturforschg. 7 b, 324 [1952].

4 E. W e i l e r, Strahlentherapie 93, 213 [1954]. 